\title{
Synovial sarcoma of anterior abdominal wall
}

\author{
Sangeetha Jayaraman · S. Devaji Rao $\cdot$ Meera Govindarajan
}

Received: 25 June 2009 / Accepted: 18 August 2009

(C) Association of Surgeons of India 2010

\begin{abstract}
Sarcomas are connective tissue tumours, and can arise in any part of the body, more commonly in the extremities. Histological types and clinical presentation of truncal sarcomas are similar to those seen in any other anatomic locations. Radiological investigations may be contributory, but biopsy is conclusive. Surgical resection with wide margins is the initial standard treatment, however a multimodal approach including radiotherapy and chemotherapy is often favoured. Because of the high recurrence rate regardless of therapy, close follow-up is imperative. We present a case of synovial sarcoma of the anterior abdominal wall.
\end{abstract}

Keywords Synovial sarcoma $\cdot$ Abdominal wall

S. Jayaraman ${ }^{1}$ S. D. Rao ${ }^{2} \cdot$ M. Govindarajan ${ }^{3}$

${ }^{1}$ Department of Surgery,

St. Isabel's Hospital, Chennai, India

${ }^{2}$ Department of Surgery,

Dhanwanthri Surgical Clinic and St. Isabel's Hospital,

Chennai, India

${ }^{3} \mathrm{R} \&$ D Histopath Lab, Chennai, India

S. D. Rao. $(\bowtie)$

E-mail: devajiraos@yahoo.co.in

\section{Case report}

A 33-year-old female presented with a painless lump in the lower abdomen right to the midline. On examination, there was a $8 \mathrm{~cm}$ diameter lump arising from the anterior rectus sheath $1 \mathrm{~cm}$ right to the midline, the lower border about $4 \mathrm{~cm}$ above the pubic bone. CT scan of the abdomen revealed it to be a mass with well defined border arising from the anterior rectus sheath (Fig. 1). She gave history of diagnostic aspiration done a year ago, which yielded altered blood, and was dismissed as a rectus sheath haematoma, and the fluid was not analysed. Presently, the tumour was attempted to be excised with a clinical diagnosis of a desmoid tumour. During surgery, the anterior rectus sheath looked intact and normal, and an incision was made $1 \mathrm{~cm}$

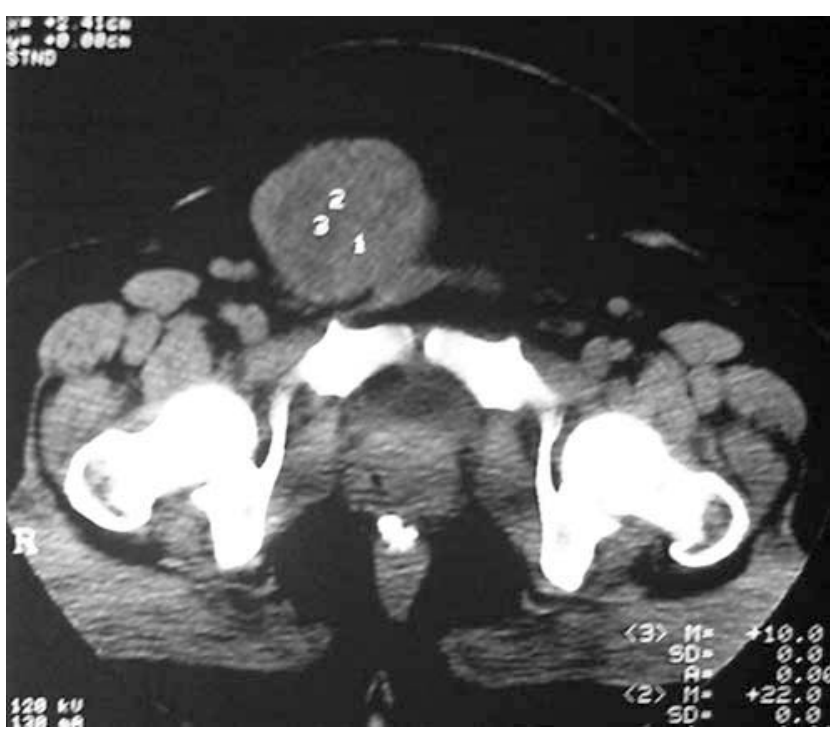

Fig. 1 CT - Well defined mass arising from anterior rectus sheath above the pubic bone 
lateral to the tumour, and the tumour was found to be soft and necrotic and had a rich vascular supply. The tumour was excised with anterior rectus sheath with wide margins; the rectus muscle was not excised as it was not infiltrated any where. The defect in the anterior rectus sheath and the external oblique aponeurosis was repaired with a polypropylene mesh, and the wound was closed with a subcutaneous drain. The histopathology of the tumour was reported as synovial sarcoma, as the immunohistochemistry showed positivity for S 100 , vimentin and cytokeratin and negativity for desmin and factor 8 . The resected margins were tumour free. Further, she was given external beam radiotherapy of $50 \mathrm{~Gy}$, and 6 cycles of ifosfamide.

\section{Discussion}

Though synovial sarcoma is a relatively common primary soft tissue malignancy, and is known to arise in an extremity, commonly in the popliteal fossa or limb girdle, occurrence at unexpected locations such as abdominal wall is rare. They occur in close association with joint capsules, tendon sheaths, bursae and facial structures. Despite the nomenclature, they rarely arise from intra-articular locations, commonly between 15 and 40 years of age, with no gender or racial or ethnic predilection.

The pathogenesis remains unclear, and it is believed that synovial sarcomas arise from primitive mesenchyme, rather than preformed synovial cells. Cytogenetic studies show $90 \%$ of cases show translocation of chromosome $\mathrm{t}(\mathrm{X} ; 18)$ (p11.2; q11.2) and fusion of SYT gene on chromosome 18 with either SSX1 (67\% of cases) or SSX2 (33\% of cases) on chromosome X, though the prognostic significance between two fusion types remains controversial [1]. Sarcomas are associated with a variety of carcinogenic agents (e.g., cholophenols, thorotrast, arsenic and radiation), and hereditary syndromes (e.g. LiFraumeni syndrome, retinoblastoma, Von Recklinghausen's disease and Gardner's syndrome).

The tumour presents as a slow growing palpable soft tissue mass with or without pain. Abdominal tumours may be associated with vague intestinal symptoms, more commonly in the lower abdomen. Metastases are present in $16-25 \%$ of patients at their initial presentation, lung being the most common site.

Radiographs show round or oval lobulated swelling of moderate density, with erosion of bone underlying it. Calcification is seen in a third of cases, which is eccentric or peripheral within the soft tissue mass. CT shows heterogenous mass with attenuation similar or slightly less than that of muscle. MRI is the optimal radiological modality for assessing the extent and characteristics of synovial sarcomas.

Generally, patients suspected of having primary soft tissue sarcomas that appear resectable do not always require preoperative biopsy [2], but when the tumour seems to involve critical structures or when neoadjuvant therapy or multidisciplinary treatment is considered, knowledge of histology and grade of tumour becomes necessary, which can be obtained only by pretreatment biopsy [3]. However, whenever biopsy is felt needed, core biopsy should be preferred which should be included in the resectable area [4]. Incisional biopsy is performed when core biopsy is insufficient or impossible [5]. Use of fine needle biopsy (FNB) has very limited value as the positive yield is extremely low. Histologically, they are divided into three types, monophasic, biphasic types and undifferentiated, the monophasic consists predominantly of spindle cells resembling fibrosarcoma, whereas, the biphasic contains epithelial cell elements in addition. Cytogenetic studies have found that biphasic tumours predominantly express SYT-SSX1 fusion transcription, while monophasic tumours express either SYT-SSX1 or SYT-SSX2 genetic rearrangement [1]. A recent immunohistochemical profile suggests that epithelial membrane antigen (EMA), cytokeratin AE1/AE3 and E-cadherin, in combination with CD34 negativity are the most useful and sensitive markers for diagnosing synovial sarcoma, more intensely of the biphasic type [6]. Poorly differentiated tumours are epithelioid in morphology with high mitotic activity and very poor prognosis.

Truncal sarcomas are staged using modified tumor, node and metastases (TNM) classification, which includes grade (GTNM). It is further subdivided to consider the location of the lesion in relationship to the superficial fascia, the lesion above the fascia as ' $a$ ' and if below as ' $b$ '. This differentiation has additional prognostic information as ' $a$ ' lesion has a better prognosis than a ' $b$ ' lesion of similar size and grade [5].

The treatment of choice is wide excision followed by adjuvant radiotherapy (external beam radiation/brachytherapy), and chemotherapy (doxorubicin). Following resection, wide defects can be reconstructed with prosthetic material and/or pedicled or free flaps.

Five years survival for intermediate to high grade sarcoma is $36-76 \%$. Local recurrence is seen in $30-50 \%$ of cases and is linked to inadequate margins and aggressive histopathology [7]. Singer et al. reported an overall survival of $67 \%$ with negative microscopic margins, compared to $49 \%$ for positive margins [8]. Metastases to lungs, lymph nodes and bones are common, majority in the first $2-5$ years of treatment.

Because local recurrence is common, close follow up of at least every 3 months for the first 2 years and then bi-annually for another 5 years is recommended. Physical examination, laboratory studies, and imaging are warranted to rule out recurrence. Chest radiographs combined with CT scanning are performed routinely bi-annually for $2-3$ years to rule out disease recurrence and to evaluate for possible pulmonary metastases. 


\section{Conclusion}

In cases of anterior abdominal wall masses, it is important to consider the possibility of a malignant tumour, synovial sarcoma.

\section{References}

1. Nagao K, Ito H, Yoshida H (1996) Chromosomal translocation $\mathrm{t}(\mathrm{X} ; 18)$ in human synovial sarcomas analysed by fluorescence in situ hybridisation using paraffin-embedded tissue. Am J Pathol 148:601-609

2. Scoggins CR, Pisters PWT (2008) Diagnosis and management of soft tissue sarcomas. Advan Surg 42:219-228
3. Hueman MT, Thornton K, Herman JM, et al. (2008) Management of extremity soft tissue sarcoma. Surg Clin N Am 88:539-557

4. Heslin MJ, Lewis JJ, Woodruff JM, et al. (1997) Core needle biopsy for diagnosis of extremity soft tissue sarcoma. Ann Surg Oncol 4:425-431

5. Jacqueline M, Wang G, Leach SD, (2008) Truncal sarcomas and abdominal desmoids. Surg Clin N Am 88:571-582

6. Vera J, Garcia MD, Marigil M, et al. (2006) Biphasic synovial sarcoma of the abdominal wall. Virchows Arch 449(3):367-372

7. Stojadinovic A, Hoos A, Karpoff HM, et al. (2001) Soft tissue sarcomas of the abdominal wall; analysis of disease pattern and treatment. Arch Surg 136:70-79

8. Singer S, Corson JM, Demetri GD, et al. (1995) Prognostic factors predictive for survivial of truncal and retroperitoneal soft tissue sarcoma. Ann Surg 221:185-195 